\title{
Stable Perturbed Iterative Algorithms for Solving New General Systems of Nonlinear Generalized Variational Inclusion in Banach Spaces
}

\author{
Ting-jian Xiong ${ }^{1}$ and Heng-you Lan ${ }^{1,2}$ \\ ${ }^{1}$ Department of Mathematics, Sichuan University of Science and Engineering, Zigong, Sichuan 643000, China \\ ${ }^{2}$ Key Laboratory Higher Education of Sichuan Province for Enterprise Informationalization and Internet of Things, Zigong, Sichuan \\ 643000, China
}

Correspondence should be addressed to Heng-you Lan; hengyoulan@163.com

Received 19 June 2014; Accepted 26 July 2014; Published 14 October 2014

Academic Editor: Jong Kyu Kim

Copyright (C) 2014 T.-j. Xiong and H.-y. Lan. This is an open access article distributed under the Creative Commons Attribution License, which permits unrestricted use, distribution, and reproduction in any medium, provided the original work is properly cited.

\begin{abstract}
We introduce and study a new general system of nonlinear variational inclusions involving generalized $m$-accretive mappings in Banach space. By using the resolvent operator technique associated with generalized $m$-accretive mappings due to Huang and Fang, we prove the existence theorem of the solution for this variational inclusion system in uniformly smooth Banach space, and discuss convergence and stability of a class of new perturbed iterative algorithms for solving the inclusion system in Banach spaces. Our results presented in this paper may be viewed as an refinement and improvement of the previously known results.
\end{abstract}

\section{Introduction}

Let $m$ be a given positive integer, for any $i \in\{1,2, \ldots, m\}, X_{i}$ a real Banach space with dual space $X_{i}^{*} . X_{i}, X_{i}^{*}$ all endowed with the norm $\|\cdot\|$, and $\langle\cdot, \cdot\rangle$ the dual pair between $X_{i}$ and $X_{i}^{*}$ (as matter of convenience). Let $2^{X_{i}}$ denote the family of all the nonempty subsets of $X_{i}, \eta_{i}: X_{i} \times X_{i} \rightarrow X_{i}^{*}$, $N_{i}: X_{1} \times X_{2} \times \cdots \times X_{m} \rightarrow X_{i}$ single-valued mappings, and $M_{i}: X_{i} \rightarrow 2^{X_{i}}$ generalized $m$-accretive mapping for $i=1,2, \ldots, m$. In this paper, we consider the following new general system for nonlinear variational inclusion involving generalized $m$-accretive mappings. Find $\left(x_{1}^{*}, x_{2}^{*}, \ldots, x_{m}^{*}\right) \in$ $X_{1} \times X_{2} \times \cdots \times X_{m}$ such that

$$
0 \in N_{i}\left(x_{1}^{*}, x_{2}^{*}, \ldots, x_{m}^{*}\right)+M_{i}\left(x_{i}^{*}\right)
$$

for all $i=1,2, \ldots, m$. Some special cases of the problem (1) had been studied by many authors. See, for example, [1-34] and the reference therein. Here, we mention some of them as follows.

Case 1. The problem (1) with $X_{i}=\mathscr{H}_{i}(i=1,2, \ldots, m)$, the Hilbert spaces, was introduced and studied as general system of monotone nonlinear variational inclusions problems by Peng and Zhao [29].

$$
\text { If } J_{q}^{-1} \eta_{i}\left(x_{i}^{1}, x_{i}^{2}\right)=x_{i}^{1}-x_{i}^{2} \text { and } M_{i}=\partial \varphi_{i}, \varphi_{i}: X_{i} \rightarrow
$$
$(-\infty,+\infty]$ is proper, convex, and lower semi-continuous functional on $X_{i}$, and $\partial \varphi_{i}$ denote the subdifferential operators of the $\varphi_{i}$ for $i=1,2, \ldots, m$, then the problem (1) is equivalent to finding $\left(x_{1}^{*}, x_{2}^{*}, \ldots, x_{m}^{*}\right) \in X_{1} \times X_{2} \times \ldots \times X_{m}$ such that

$$
\begin{aligned}
& \left\langle N_{i}\left(x_{1}^{*}, x_{2}^{*}, \ldots, x_{m}^{*}\right), j\left(x_{i}-x_{i}^{*}\right)\right\rangle \\
& \quad \geq \rho_{i}\left(\varphi_{i}\left(x_{i}^{*}\right)-\varphi_{i}\left(x_{i}\right)\right), \quad \forall x_{i} \in X_{i} .
\end{aligned}
$$

When $X_{i}=X, 2$-uniformly smooth Banach space with the smooth constant $K, C$ is a nonempty closed convex subset of $X, N_{i}\left(x_{1}, x_{2}, \ldots, x_{m}\right)=\rho_{i} A_{i}\left(x_{i+1}\right)+x_{i}-x_{i+1}$, where $A_{i}: C \rightarrow X$ and $\rho_{i}>0$ and $x_{m+1}=x_{1}$ for $i=1,2, \ldots, m$; the problem (2) reduces to the following system of finding $\left(x_{1}^{*}, x_{2}^{*}, \ldots, x_{m}^{*}\right) \in C \times C \times \cdots \times C$ such that

$$
\begin{aligned}
& \left\langle\rho_{i} A_{i}\left(x_{i+1}^{*}\right)+x_{i}^{*}-x_{i+1}^{*}, j\left(x-x_{i}^{*}\right)\right\rangle \\
& \quad \geq \rho_{i}\left(\varphi_{i}\left(x_{i}^{*}\right)-\varphi_{i}(x)\right), \quad \forall x \in X .
\end{aligned}
$$


Further, in the problem (3), when $\varphi_{i}$ is the indicator function of a nonempty closed convex set $C$, in $X$ defined by

$$
\varphi_{i}(y)= \begin{cases}0, & y \in C, \\ +\infty, & y \notin C,\end{cases}
$$

then the system $(3)$ reduces to finding $\left(x_{1}^{*}, x_{2}^{*}, \ldots, x_{m}^{*}\right) \in C \times$ $C \times \cdots \times C$ such that

$$
\begin{array}{cc}
\left\langle\rho_{1} A_{1} x_{2}^{*}+x_{1}^{*}-x_{2}^{*}, j\left(x-x_{1}^{*}\right)\right\rangle \geq 0, & \forall x \in C, \\
\left\langle\rho_{2} A_{2} x_{3}^{*}+x_{2}^{*}-x_{3}^{*}, j\left(x-x_{2}^{*}\right)\right\rangle \geq 0, & \forall x \in C, \\
\left\langle\rho_{3} A_{3} x_{4}^{*}+x_{3}^{*}-x_{4}^{*}, j\left(x-x_{3}^{*}\right)\right\rangle \geq 0, & \forall x \in C, \\
\cdots & \\
\left\langle\rho_{m} A_{m} x_{1}^{*}+x_{m}^{*}-x_{1}^{*}, j\left(x-x_{m}^{*}\right)\right\rangle \geq 0, & \forall x \in C,
\end{array}
$$

which was introduced and studied by Zhu et al. [34].

Case 2. If $m=3$, then the system (3) is equivalent to finding $\left(x_{1}^{*}, x_{2}^{*}, x_{3}^{*}\right) \in C \times C \times C$ such that

$$
\begin{aligned}
& \left\langle\rho_{1} A_{1} x_{2}^{*}+x_{1}^{*}-x_{2}^{*}, j\left(x-x_{1}^{*}\right)\right\rangle \\
& \quad \geq \rho_{1}\left(\varphi_{1}\left(x_{1}^{*}\right)-\varphi_{1}(x)\right), \quad \forall x \in C, \\
& \left\langle\rho_{2} A_{2} x_{3}^{*}+x_{2}^{*}-x_{3}^{*}, j\left(x-x_{2}^{*}\right)\right\rangle \\
& \quad \geq \rho_{2}\left(\varphi_{2}\left(x_{2}^{*}\right)-\varphi_{2}(x)\right), \quad \forall x \in C, \\
& \left\langle\rho_{3} A_{3} x_{1}^{*}+x_{3}^{*}-x_{1}^{*}, j\left(x-x_{3}^{*}\right)\right\rangle \\
& \geq \rho_{3}\left(\varphi_{3}\left(x_{3}^{*}\right)-\varphi_{3}(x)\right), \quad \forall x \in C .
\end{aligned}
$$

It is easy to see that the mathematical model studied by Saewan and Kumam [31] is a variant of (6).

Case 3. If $m=2$, then the problem (1) reduces to find $\left(x^{*}, y^{*}\right) \in X_{1} \times X_{2}$ such that

$$
0 \in N_{1}\left(x^{*}, y^{*}\right)+M_{1}\left(x^{*}\right), \quad 0 \in N_{2}\left(x^{*}, y^{*}\right)+M_{2}\left(y^{*}\right) \text {. }
$$

Problem (7) is called a system of strongly nonlinear quasivariational inclusion involving generalized $m$-accretive mappings, it is considered and studied by Lan [19]. There are many special cases of the problems (7) that can be found in $[3,7,12-$ $14,17,20,28,30]$ and the references cited therein.

Case 4. If $m=1$ and $X_{1}=\mathscr{H}$, then the problem (1) reduces to finding $x^{*} \in \mathscr{H}$ such that

$$
0 \in N\left(x^{*}\right)+M\left(x^{*}\right)
$$

which was introduced and studied by Fang and Huang [8]. We remark that for appropriate and suitable choices of positive integer $m$, the mappings $\eta_{i}, N_{i}$, and $M_{i}$, and the spaces $X_{i}$ for $i=1,2, \ldots, m$, one can know that the problem (1) includes a number of general class of variational character known problems, including minimization or maximization (whether constraint or not) of functions and minimax problems et al. as special cases. For more details, see [1-34] and the reference therein.
On the other hand, many authors discussed stability of the iterative sequence generated by the algorithm for solving the problems that they studied. Lan [19] introduced the notion of $S$-stable or stable with respect to $S$. Moreover, Agarwal et al. [1, 2], Jin [16], Kazmi and Bhat [18], and Lan and Kim [21] constructed some stability under suitable conditions, respectively.

Motivated and inspired by the above works, the main purpose of this paper is to introduce and study the new general system of nonlinear variational inclusions (1) involving generalized $m$-accretive mapping in uniformly smooth Banach spaces. By using the resolvent operator technique for generalized $m$-accretive, we prove the existence theorem of the solution for this kind of system of variational inclusions in Banach spaces and discuss the convergence and stability of a new perturbed iterative algorithm for solving this general system of nonlinear variational inclusions in Banach spaces.

\section{Preliminaries}

In order to get the main results of the paper, we need the following concepts and lemmas. Let $X$ be a real Banach space with dual space $X^{*},\langle\cdot, \cdot\rangle$ the dual pair between $X$ and $X^{*}$, and $2^{X}$ denote the family of all the nonempty subsets of $X$. The generalized duality mapping $J_{q}: X \rightarrow 2^{X^{*}}$ is defined by

$$
\begin{array}{r}
J_{q}(x)=\left\{f^{*} \in X^{*}:\left\langle x, f^{*}\right\rangle=\|x\|^{q},\left\|f^{*}\right\|=\|x\|^{q-1}\right\}, \\
\forall x \in X,
\end{array}
$$

where $q>1$ is a constant. In particular, $J_{2}$ is the usual normalized duality mapping. It is known that if $X^{*}$ is strictly convex or $X$ is a uniformly smooth Banach space, then $J_{q}$ is single-valued (see [33]), and if $X=\mathscr{H}$, the Hilbert space, then $\mathrm{J}_{2}$ becomes the identity mapping on $\mathscr{H}$. We will denote the single-valued duality mapping by $j_{q}$.

In order to construct convergence and stability for researching the problem (1), we need to be using the following definition and lemma.

Definition 1. Let $X_{i}$ be Banach spaces, and let $N_{i}: X_{1} \times X_{2} \times$ $\cdots \times X_{m} \rightarrow X_{i}$ be single mappings for $(i=1,2, \ldots, m)$. Then $N_{i}$ is said to be

(i) $\sigma_{j}$-strongly accretive with respect to $j$ th argument if for any $\left(x_{1}, \ldots, x_{j-1}, x_{j}^{1}, x_{j+1}, \ldots, x_{m}\right)$, $\left(x_{1}, \ldots, x_{j-1}, x_{j}^{2}, x_{j+1}, \ldots, x_{m}\right) \in X_{1} \times X_{2} \times \cdots \times X_{m}$, there exists $j_{q_{j}}\left(x_{j}^{1}-x_{j}^{2}\right) \in J_{q_{j}}\left(x_{j}^{1}-x_{j}^{2}\right)$, such that

$$
\begin{gathered}
\left\langle N_{i}\left(x_{1}, \ldots, x_{j-1}, x_{j}^{1}, x_{j+1}, \ldots, x_{m}\right)\right. \\
-N_{i}\left(x_{1}, \ldots, x_{j-1}, x_{j}^{2}, x_{j+1}, \ldots, x_{m}\right), \\
\left.j_{q_{j}}\left(x_{j}^{1}-x_{j}^{2}\right)\right\rangle \geq \sigma_{j}\left\|x_{j}^{1}-x_{j}^{2}\right\|^{q_{j}},
\end{gathered}
$$

where $q_{j}>1$ is a constant; 
(ii) $\left(\zeta_{i 1}, \ldots, \zeta_{i j}, \ldots, \zeta_{i m}\right)$-Lipschitz continuous if there exists constants $\zeta_{i 1}>0, \ldots, \zeta_{i j}>0, \ldots, \zeta_{i m}>0$, such that

$$
\begin{aligned}
& \left\|N_{i}\left(x_{1}, \ldots, x_{j}, \ldots, x_{m}\right)-N_{i}\left(y_{1}, \ldots, y_{j}, \ldots, y_{m}\right)\right\| \\
& \quad \leq \sum_{j=1}^{m} \zeta_{i j}\left\|x_{j}-y_{j}\right\|,
\end{aligned}
$$

for all $x_{j}, y_{j} \in X_{j}$ and $j=1,2 \ldots, m$.

Remark 2. When $X_{i}=\mathscr{H}_{i}(i=1,2, \ldots, m), \mathscr{H}_{i}$ is different or the same as Hilbert spaces, (i) and (ii) in Definition 1 reduce to strongly monotonicity with respect to $j$ th argument of $N_{i}$ and $\left(\zeta_{i 1}, \ldots, \zeta_{i j}, \ldots, \zeta_{i m}\right)$-Lipschitz continuity of $N_{i}$, respectively (see [29]).

Definition 3. Let $\eta: X \times X \rightarrow X^{*}$ be single-valued mapping. Then set-valued mapping $M: X \rightarrow 2^{X}$ is said to be

(i) accretive if

$$
\begin{array}{r}
\left\langle u-v, J_{q}(x-y)\right\rangle \geq 0, \quad \forall x, y \in X, u \in M(x), \\
v \in M(y) ;
\end{array}
$$

(ii) $\eta$-accretive if

$\langle u-v, \eta(x, y)\rangle \geq 0, \quad \forall x, y \in X, u \in M(x), v \in M(y) ;$

(iii) $m$-accretive if $M$ is accretive and $(I+\rho M)(X)=X$ for all $\rho>0$, where $I$ denotes the identity operator on $X$;

(iv) generalized $m$-accretive if $M$ is $\eta$-accretive and $(I+$ $\rho M)(X)=X$ for all $\rho>0$.

Remark 4. When $X=X^{*}=\mathscr{H}$, (i)-(iv) of Definition 3 reduce to the definitions of monotone operators, $\eta$-monotone operators, classical maximal monotone operators, and maximal $\eta$-monotone operators; if $\eta(x, y)=J_{2}(x-y)$, then (ii) and (iv) of Definition 3 reduce to the definitions of accretive and $m$-accretive of uniformly smooth Banach spaces (see $[10,11])$.

Definition 5. The mapping $\eta: X \times X \rightarrow X^{*}$ is said to be

(i) $\delta$-strongly monotone if there exists a constant $\delta>0$ such that

$$
\left\langle x^{1}-x^{2}, \eta\left(x^{1}, x^{2}\right)\right\rangle \geq \delta\left\|x^{1}-x^{2}\right\|^{2}, \quad \forall x^{1}, x^{2} \in X
$$

(ii) $\tau$-Lipschitz continuous if there exists a constant $\tau>0$ such that

$$
\left\|\eta\left(x^{1}, x^{2}\right)\right\| \leq \tau\left\|x^{1}-x^{2}\right\|, \quad \forall x^{1}, x^{2} \in X .
$$

In [10], Huang and Fang show that for any $\rho_{i}>0$, inverse mapping $\left(I+\rho_{i} M_{i}\right)^{-1}$ is single-valued, if $\eta_{i}: X_{i} \times X_{i} \rightarrow X_{i}^{*}$ is strict monotone and $M_{i}: X_{i} \rightarrow 2^{X_{i}}$ is generalized $m$ accretive mapping, where $I$ is the identity mapping. Based on this fact, Huang and Fang [10] gave the following definition.
Definition 6. Let $\eta_{i}: X_{i} \times X_{i} \rightarrow X_{i}^{*}$ be strictly monotone mapping, and let $M_{i}: X_{i} \rightarrow 2^{X_{i}}$ be generalized $m$-accretive mapping. Then the resolvent $J_{M_{i}}^{\rho_{i}}$ for $M_{i}$ is defined as follows:

$$
J_{M_{i}}^{\rho_{i}}\left(x_{i}\right)=\left(I+\rho_{i} M_{i}\right)^{-1}\left(x_{i}\right), \quad \forall x_{i} \in X_{i},
$$

where $\rho_{i}>0$ is a constant and $I$ denotes the identity mapping on $X_{i}$ for $i=1,2, \ldots, m$.

Lemma 7 (see $[10,11])$. Let $\eta_{i}: X_{i} \times X_{i} \rightarrow X_{i}^{*}$ be $\tau_{i}$-Lipschitz continuous and $\delta_{i}$-strongly monotone, and let $M_{i}: X_{i} \rightarrow 2_{i}^{X}$ be generalized m-accretive mapping. Then for any $\rho_{i}>0$, the resolvent operator $J_{M_{i}}^{\rho_{i}}$ for $M_{i}$ is $\tau_{i} / \delta_{i}$-Lipschitz continuous; that is,

$$
\begin{aligned}
& \left\|J_{M_{i}}^{\rho_{i}}\left(x_{i}\right)-J_{M_{i}}^{\rho_{i}}\left(y_{i}\right)\right\| \\
& \quad \leq \frac{\tau_{i}}{\delta_{i}}\left\|x_{i}-y_{i}\right\|, \quad \forall x_{i}, y_{i} \in X_{i}, i=1,2, \ldots, m .
\end{aligned}
$$

The modules of smoothness is a measure, it is depicted geometric structure of the underlying Banach space. The modules of smoothness of Banach space $X$ are the function $\rho_{X}:[0,+\infty) \rightarrow[0,+\infty)$ defined by

$$
\rho_{X}(t)=\sup \left\{\frac{1}{2}(\|x+y\|+\|x-y\|)-1:\|x\| \leq 1,\|y\| \leq t\right\} .
$$

A Banach space $X$ is called uniformly smooth if $\lim _{t \rightarrow 0}\left(\rho_{X}(t) / t\right)=0 . X$ is called $q$-uniformly smooth if there exists a constant $c>0$ such that $\rho_{X}(t) \leq c t^{q}$, where $q>1$ is a real number.

Remark that $J_{q}$ is single-valued if $X$ is uniformly smooth, and Hilbert space and $L_{p}\left(\right.$ or $\left.l_{p}\right)(2 \leq p<+\infty)$ spaces are 2-uniformly smooth Banach spaces. In what follows, we will denote the single-valued generalized duality mapping by $j_{q}$.

In the study of characteristic inequalities in $q$-uniformly smooth Banach spaces, $\mathrm{Xu}$ [35] proved the following result.

Lemma 8. Let $q>1$ be a given real number and let $X$ be $a$ real uniformly smooth Banach space. Then $X$ is q-uniformly smooth if and only if there exists a constant $c_{q}>0$ such that for all $x, y \in X, j_{q}(x) \in J_{q}(x)$, there holds the following inequality:

$$
\|x+y\|^{q} \leq\|x\|^{q}+q\left\langle y, j_{q}(x)\right\rangle+c_{q}\|y\|^{q} .
$$

Definition 9. Let $S$ be a self-map of $X, x_{0} \in X$, and let $x_{n+1}=$ $h\left(S, x_{n}\right)$ define an iteration procedure which yields a sequence of points $\left\{x_{n}\right\}_{n=0}^{\infty}$ in $X$. Suppose that $\{x \in X: S x=x\} \neq \emptyset$ and $\left\{x_{n}\right\}_{n=0}^{\infty}$ converges to a fixed point $x^{*}$ of $S$. Let $\left\{u_{n}\right\} \subset X$ and let $\epsilon_{n}=\left\|u_{n+1}-h\left(S, u_{n}\right)\right\|$. If $\lim _{n \rightarrow \infty} \epsilon_{n}=0$ implies that $u_{n} \rightarrow x^{*}$, then the iteration procedure defined by $x_{n+1}=h\left(S, x_{n}\right)$ is said to be $S$-stable or stable with respect to $S$.

Lemma 10 (see [36]). Let $\left\{a_{n}\right\},\left\{b_{n}\right\}$, and $\left\{c_{n}\right\}$ be three nonnegative real sequences satisfying the following condition: there exists a natural number $n_{0}$ such that

$$
a_{n+1} \leq\left(1-t_{n}\right) a_{n}+b_{n} t_{n}+c_{n}, \quad \forall n \geq n_{0},
$$

where $t_{n} \in[0,1], \sum_{n=0}^{\infty} t_{n}=+\infty, \lim _{n \rightarrow \infty} b_{n}=0$, and $\sum_{n=0}^{\infty} c_{n}<+\infty$. Then $a_{n}$ converges to 0 as $n \rightarrow \infty$. 


\section{Existence Theorem}

In this section, we will give the existence theorem of the problem (1). The solvability of the problem (1) depends on the equivalence between (1) and the problem of finding the fixed point of the associated generalized resolvent operator. It follows from the definition of generalized resolvent operator $J_{M_{i}}^{\rho_{i}}(i=1,2, \ldots, m)$ that we can obtain the following conclusion.

Lemma 11. Let $\eta_{i}: X_{i} \times X_{i} \rightarrow X_{i}^{*}, N_{i}: X_{1} \times X_{2} \times \cdots \times X_{m} \rightarrow$ $X_{i}$ single-valued mappings, and $M_{i}: X_{i} \rightarrow 2^{X_{i}}$ generalized $m$-accretive mapping for $(i=1,2, \ldots, m)$. Then the following statements are mutually equivalent.

(i) An element $\left(x_{1}^{*}, x_{2}^{*}, \ldots, x_{m}^{*}\right) \in X_{1} \times X_{2} \times \cdots \times X_{m}$ is a solution to the problem (1).

(ii) There is an $\left(x_{1}^{*}, x_{2}^{*}, \ldots, x_{m}^{*}\right) \in X_{1} \times X_{2} \times \cdots \times X_{m}$ such that

$$
x_{i}^{*}=J_{M_{i}}^{\rho_{i}}\left[x_{i}^{*}-\rho_{i} N_{i}\left(x_{1}^{*}, \ldots, x_{i-1}^{*}, x_{i}^{*}, x_{i+1}^{*}, \ldots, x_{m}^{*}\right)\right] \text {, }
$$

where $J_{M_{i}}^{\rho_{i}}=\left(I+\rho_{i} M_{i}\right)^{-1}$, and $\rho_{i}>0$ is constants for all $i=1,2, \ldots, m$.

(iii) For any given constants $\rho_{i}>0$, the map $F: X_{1} \times X_{2} \times$ $\cdots \times X_{m} \rightarrow X_{1} \times X_{2} \times \cdots \times X_{m}$ is defined by

$$
\begin{gathered}
F\left(u_{1}, u_{2}, \ldots, u_{m}\right) \\
=\left(P_{\rho_{1}}\left(u_{1}, u_{2}, \ldots, u_{m}\right), \ldots, P_{\rho_{i}}\left(u_{1}, u_{2}, \ldots, u_{m}\right), \ldots,\right. \\
\left.\quad P_{\rho_{m}}\left(u_{1}, u_{2}, \ldots, u_{m}\right)\right)
\end{gathered}
$$

for all $u_{i} \in X_{i}$ and $i=1,2, \ldots, m$, has a fixed point $\left(x_{1}^{*}, x_{2}^{*}, \ldots, x_{m}^{*}\right) \in X_{1} \times X_{2} \times \cdots \times X_{m}$, where maps $P_{\rho_{i}}: X_{1} \times X_{2} \times \cdots \times X_{m} \rightarrow X_{i}$ are defined by

$$
\begin{aligned}
P_{\rho_{i}}\left(u_{1}, u_{2}, \ldots, u_{m}\right) & \\
& =J_{M_{i}}^{\rho_{i}}\left[u_{i}-\rho_{i} N_{i}\left(u_{1}, \ldots, u_{i-1}, u_{i}, u_{i+1}, \ldots, u_{m}\right)\right]
\end{aligned}
$$

for $u_{i} \in X_{i}$ and $i=1,2, \ldots, m$.

Proof. We first prove that (i) $\Leftrightarrow$ (ii). Let $\left(x_{1}^{*}, x_{2}^{*}, \ldots, x_{m}^{*}\right) \in$ $X_{1} \times X_{2} \times \cdots \times X_{m}$ satisfy the relation in (ii). Then, the definition of resolvent operator $J_{M_{i}}^{\rho_{i}}$ implies that this equality holds if and only if

$$
x_{i}^{*}-\rho_{i} N_{i}\left(x_{1}^{*}, \ldots, x_{i-1}^{*}, x_{i}^{*}, x_{i+1}^{*}, \ldots, x_{m}^{*}\right) \in\left(I+\rho_{i} M_{i}\right)\left(x_{i}^{*}\right)
$$

for $i=1,2, \ldots, m$; that is

$$
0 \in N_{i}\left(x_{1}^{*}, x_{2}^{*}, \ldots, x_{m}^{*}\right)+M_{i}\left(x_{i}^{*}\right),
$$

where $i=1,2, \ldots, m$. Thus $\left(x_{1}^{*}, x_{2}^{*}, \ldots, x_{m}^{*}\right) \in X_{1} \times X_{2} \times \cdots \times$ $X_{m}$ is the solution of the problem (1).
Next, we show (ii) $\Leftrightarrow$ (iii). If $\left(x_{1}^{*}, x_{2}^{*}, \ldots, x_{m}^{*}\right) \in X_{1} \times X_{2} \times$ $\cdots \times X_{m}$ satisfy following relation:

$$
x_{i}^{*}=J_{M_{i}}^{\rho_{i}}\left[x_{i}^{*}-\rho_{i} N_{i}\left(x_{1}^{*}, \ldots, x_{i-1}^{*}, x_{i}^{*}, x_{i+1}^{*}, \ldots, x_{m}^{*}\right)\right] \text {, }
$$

then, for any $i=1,2, \ldots, m$, it follows from

$$
\begin{aligned}
P_{\rho_{i}}( & \left.x_{1}^{*}, x_{2}^{*}, \ldots, x_{m}^{*}\right) \\
& =J_{M_{i}}^{\rho_{i}}\left[x_{i}^{*}-\rho_{i} N_{i}\left(x_{1}^{*}, \ldots, x_{i-1}^{*}, x_{i}^{*}, x_{i+1}^{*}, \ldots, x_{m}^{*}\right)\right]
\end{aligned}
$$

that

$$
P_{\rho_{i}}\left(x_{1}^{*}, x_{2}^{*}, \ldots, x_{m}^{*}\right)=x_{i}^{*} .
$$

Hence, $\left(x_{1}^{*}, x_{2}^{*}, \ldots, x_{m}^{*}\right) \in X_{1} \times X_{2} \times \cdots \times X_{m}$ is a fixed point of the mapping

$$
\begin{gathered}
F\left(u_{1}, u_{2}, \ldots, u_{m}\right) \\
=\left(P_{\rho_{1}}\left(u_{1}, u_{2}, \ldots, u_{m}\right), \ldots, P_{\rho_{i}}\left(u_{1}, u_{2}, \ldots, u_{m}\right), \ldots,\right. \\
\left.\quad P_{\rho_{m}}\left(u_{1}, u_{2}, \ldots, u_{m}\right)\right) .
\end{gathered}
$$

Conversely, if $\left(x_{1}^{*}, x_{2}^{*}, \ldots, x_{m}^{*}\right) \in X_{1} \times X_{2} \times \cdots \times X_{m}$ is a fixed point of the mapping $F: X_{1} \times X_{2} \times \cdots \times X_{m} \rightarrow X_{1} \times X_{2} \times$ $\cdots \times X_{m}$, then

$$
P_{\rho_{i}}\left(x_{1}^{*}, x_{2}^{*}, \ldots, x_{m}^{*}\right)=x_{i}^{*}
$$

for $i=1,2, \ldots, m$. Hence, from

$$
\begin{aligned}
P_{\rho_{i}}( & \left.x_{1}^{*}, x_{2}^{*}, \ldots, x_{m}^{*}\right) \\
& =J_{M_{i}}^{\rho_{i}}\left[x_{i}^{*}-\rho_{i} N_{i}\left(x_{1}^{*}, \ldots, x_{i-1}^{*}, x_{i}^{*}, x_{i+1}^{*}, \ldots, x_{m}^{*}\right)\right],
\end{aligned}
$$

we have

$$
x_{i}^{*}=J_{M_{i}}^{\rho_{i}}\left[x_{i}^{*}-\rho_{i} N_{i}\left(x_{1}^{*}, \ldots, x_{i-1}^{*}, x_{i}^{*}, x_{i+1}^{*}, \ldots, x_{m}^{*}\right)\right]
$$

for $i=1,2, \ldots, m$. Therefore $\left(x_{1}^{*}, x_{2}^{*}, \ldots, x_{m}^{*}\right) \in X_{1} \times X_{2} \times$ $\cdots \times X_{m}$ satisfy the relation of (ii).

Theorem 12. Let $X_{i}$ be a real $q_{i}$-uniformly smooth Banach space with $q_{i}>1$ and let $\eta_{i}: X_{i} \times X_{i} \rightarrow X_{i}^{*}$ be $\tau_{i}$-Lipschitz continuous and $\delta_{i}$-strongly monotone for any $i=1,2, \ldots, m$. Suppose that $M_{i}: X_{i} \rightarrow 2^{X_{i}}$ is generalized $m$-accretive mapping, and $N_{i}: X_{1} \times X_{2} \times \cdots \times X_{m} \rightarrow X_{i}$ is $\sigma_{i}$-strongly accretive in the ith argument and $\left(\zeta_{i 1}, \ldots, \zeta_{i i}, \ldots, \zeta_{i m}\right)$ Lipschitz continuous for $i=1,2, \ldots, m$. If

$$
\frac{\tau_{j}}{\delta_{j}} \sqrt[q_{j}]{1-q_{j} \rho_{j} \sigma_{j}+c_{q_{j}} \rho_{j}^{q_{j}} \zeta_{j j}^{q_{j}}}+\sum_{i=1, i \neq j}^{m} \frac{\zeta_{i j} \rho_{i} \tau_{i}}{\delta_{i}}<1,
$$

where $c_{q_{j}}$ is the constants as in Lemma 8 for $j=1,2, \ldots, m$, then problem (1) has a unique solution $\left(x_{1}^{*}, x_{2}^{*}, \ldots, x_{m}^{*}\right) \in$ $X_{1} \times X_{2} \times \cdots \times X_{m}$. 
Proof. For any given $\rho_{i}>0$ and $i=1,2, \ldots, m$, we first define $P_{\rho_{i}}: X_{1} \times X_{2} \times \cdots \times X_{m} \rightarrow X_{i}$ as follows:

$$
P_{\rho_{i}}\left(u_{1}, u_{2}, \ldots, u_{m}\right)=J_{M_{i}}^{\rho_{i}}\left[u_{i}-\rho_{i} N_{i}\left(u_{1}, u_{2}, \ldots, u_{m}\right)\right],
$$

for all $u_{i} \in X_{i}$. Now define $\|\cdot\|_{*}$ on $X_{1} \times X_{2} \times \cdots \times X_{m}$ by

$$
\begin{aligned}
& \left\|\left(u_{1}, u_{2}, \ldots, u_{m}\right)\right\|_{*}=\sum_{i=1}^{m}\left\|u_{i}\right\|, \\
& \forall\left(u_{1}, u_{2}, \ldots, u_{m}\right) \in X_{1} \times X_{2} \times \cdots \times X_{m} .
\end{aligned}
$$

It is easy to see that $\left(X_{1} \times X_{2} \times \cdots \times X_{m},\|\cdot\|_{*}\right)$ is a Banach space. In fact

(i) $\left\|\left(u_{1}, u_{2}, \ldots, u_{m}\right)\right\|_{*}=\sum_{i=1}^{m}\left\|u_{i}\right\| \geq 0$, the negative being satisfied;

(ii) for all real number $\alpha$,

$$
\begin{aligned}
\left\|\alpha\left(u_{1}, u_{2}, \ldots, u_{m}\right)\right\|_{*} & =\left\|\left(\alpha u_{1}, \alpha u_{2}, \ldots, \alpha u_{m}\right)\right\|_{*} \\
& =\sum_{i=1}^{m}\left\|\alpha u_{i}\right\|=\sum_{i=1}^{m}|\alpha|\left\|u_{i}\right\| \\
& =|\alpha| \sum_{i=1}^{m}\left\|u_{i}\right\|=|\alpha|\left\|\left(u_{1}, u_{2}, \ldots, u_{m}\right)\right\|_{*}
\end{aligned}
$$

homogeneity being satisfied;

(iii) for all $\left(u_{1}, u_{2}, \ldots, u_{m}\right),\left(v_{1}, v_{2}, \ldots, v_{m}\right) \in X_{1} \times X_{2} \times \cdots \times$ $X_{m}$

$$
\begin{aligned}
& \left\|\left(u_{1}, u_{2}, \ldots, u_{m}\right)+\left(v_{1}, v_{2}, \ldots, v_{m}\right)\right\|_{*} \\
& =\left\|\left(u_{1}+v_{1}, u_{2}+v_{2}, \ldots, u_{m}+v_{m}\right)\right\|_{*} \\
& =\sum_{i=1}^{m}\left\|u_{i}+v_{i}\right\| \leq \sum_{i=1}^{m}\left(\left\|u_{i}\right\|+\left\|v_{i}\right\|\right) \\
& =\sum_{i=1}^{m}\left\|u_{i}\right\|+\sum_{i=1}^{m}\left\|v_{i}\right\| \\
& =\left\|\left(u_{1}, u_{2}, \ldots, u_{m}\right)\right\|_{*}+\left\|\left(v_{1}, v_{2}, \ldots, v_{m}\right)\right\|_{*},
\end{aligned}
$$

the triangle inequality being satisfied;

(iv) let $\left\|\left(u_{1}, u_{2}, \ldots, u_{m}\right)\right\|_{*}=0$; that is, $\sum_{i=1}^{m}\left\|u_{i}\right\|=0$; this implies that $\left\|u_{i}\right\|=0(i=1,2, \ldots, m)$; thus $u_{i}=0(i=$ $1,2, \ldots, m)$; we get $\|\cdot\|_{*}$ is a norm on the $X_{1} \times X_{2} \times$ $\cdots \times X_{m}$

(v) let $\left(u_{1}^{n}, u_{2}^{n}, \ldots, u_{m}^{n}\right) \in X_{1} \times X_{2} \times \cdots \times X_{m}$ is Cauchy sequence; that is, for $\forall \epsilon>0$, there exists a positive integer $N$; let $n>N$; we have

$$
\begin{gathered}
\left\|\left(u_{1}^{n+p}, u_{2}^{n+p}, \ldots, u_{m}^{n+p}\right)-\left(u_{1}^{n}, u_{2}^{n}, \ldots, u_{m}^{n}\right)\right\|_{*} \\
=\sum_{i=1}^{m}\left\|u_{i}^{n+p}-u_{i}^{n}\right\|<\epsilon .
\end{gathered}
$$

Thus, for all $i \in\{1,2,3, \ldots, m\}$, we have $\left\|u_{i}^{n+p}-u_{i}^{n}\right\|<$ $\epsilon(n>N, p=1,2,3, \ldots)$; that is, $\left\{u_{i}^{n}\right\} \subset X_{i}$ is also Cauchy sequence; thus $\lim _{n \rightarrow \infty} u_{i}^{n}=u_{i} \in X_{i}$ for $i=$ $1,2, \ldots, m$; we get $\left(u_{1}, u_{2}, \ldots, u_{m}\right) \in X_{1} \times X_{2} \times \cdots \times X_{m}$ and $\left(u_{1}, u_{2}, \ldots, u_{m}\right)$ is a cluster point on the $\left(X_{1} \times X_{2} \times\right.$ $\left.\cdots \times X_{m},\|\cdot\|_{*}\right)$; we claim $\left(X_{1} \times X_{2} \times \cdots \times X_{m},\|\cdot\|_{*}\right)$ is a Banach space.

Now, by (34), for any given $\rho_{i}>0$, define mapping $F$ : $X_{1} \times X_{2} \times \cdots \times X_{m} \rightarrow X_{1} \times X_{2} \times \cdots \times X_{m}$ by

$$
\begin{aligned}
& F\left(u_{1}, u_{2}, \ldots, u_{m}\right) \\
& =\left(P_{\rho_{1}}\left(u_{1}, u_{2}, \ldots, u_{m}\right), \ldots, P_{\rho_{i}}\left(u_{1}, u_{2}, \ldots, u_{m}\right), \ldots,\right. \\
& \left.\quad P_{\rho_{m}}\left(u_{1}, u_{2}, \ldots, u_{m}\right)\right),
\end{aligned}
$$

where $u_{i} \in X_{i}$ for $i=1,2, \ldots, m$.

In the sequel, we prove that $F$ is a contractive mapping on the $\left(X_{1} \times X_{2} \times \cdots \times X_{m},\|\cdot\|_{*}\right)$. In fact, for any $u_{i}, v_{i} \in X_{i}$ and $i=1,2, \ldots, m$, it follows from (34) and Lemma 7 that

$$
\begin{aligned}
& \left\|P_{\rho_{i}}\left(u_{1}, u_{2}, \ldots, u_{m}\right)-P_{\rho_{i}}\left(v_{1}, v_{2}, \ldots, v_{m}\right)\right\| \\
& =\| J_{M_{i}}^{\rho_{i}}\left[u_{i}-\rho_{i} N_{i}\left(u_{1}, u_{2}, \ldots, u_{m}\right)\right] \\
& \quad-J_{M_{i}}^{\rho_{i}}\left[v_{i}-\rho_{i} N_{i}\left(v_{1}, v_{2}, \ldots, v_{m}\right)\right] \| \\
& \leq \frac{\tau_{i}}{\delta_{i}}\left\|u_{i}-v_{i}-\rho_{i}\left(N_{i}\left(u_{1}, u_{2}, \ldots, u_{m}\right)-N_{i}\left(v_{1}, v_{2}, \ldots, v_{m}\right)\right)\right\| \\
& \leq \frac{\tau_{i}}{\delta_{i}} \| u_{i}-v_{i} \\
& \quad-\rho_{i}\left(N_{i}\left(u_{1}, \ldots, u_{i-1}, u_{i}, u_{i+1}, \ldots, u_{m}\right)\right. \\
& \quad-\frac{\rho_{i} \tau_{i}}{\delta_{i}} \| N_{i}\left(u_{1}, \ldots, u_{i-1}, v_{i}, u_{i+1}, \ldots, u_{m}\right) \\
& \quad-N_{i}\left(v_{1}, \ldots, v_{i-1}, v_{i}, v_{i+1}, \ldots, v_{m}\right) \| .
\end{aligned}
$$

By assumptions and Lemma 8, we have

$$
\begin{aligned}
& \| u_{i}-v_{i}-\rho_{i}\left(N_{i}\left(u_{1}, \ldots, u_{i-1}, u_{i}, u_{i+1}, \ldots, u_{m}\right)\right. \\
& \left.-N_{i}\left(u_{1}, \ldots, u_{i-1}, v_{i}, u_{i+1}, \ldots, u_{m}\right)\right) \|^{q_{i}} \\
& \leq\left\|u_{i}-v_{i}\right\|^{q_{i}} \\
& +c_{q_{i}} \rho_{i}^{q_{i}} \| N_{i}\left(u_{1}, \ldots, u_{i-1}, u_{i}, u_{i+1}, \ldots, u_{m}\right) \\
& -N_{i}\left(u_{1}, \ldots, u_{i-1}, v_{i}, u_{i+1}, \ldots, u_{m}\right) \|^{q_{i}} \\
& -q_{i} \rho_{i}\left\langle N_{i}\left(u_{1}, \ldots, u_{i-1}, u_{i}, u_{i+1}, \ldots, u_{m}\right)\right. \\
& \left.-N_{i}\left(u_{1}, \ldots, u_{i-1}, v_{i}, u_{i+1}, \ldots, u_{m}\right), j_{q_{i}}\left(u_{i}-v_{i}\right)\right\rangle \\
& \leq\left(1-q_{i} \rho_{i} \sigma_{i}+c_{q_{i}} \rho_{i}^{q_{i}} \zeta_{i i}^{q_{i}}\right)\left\|u_{i}-v_{i}\right\|^{q_{i}},
\end{aligned}
$$




$$
\begin{aligned}
\| & N_{i}\left(u_{1}, \ldots, u_{i-1}, v_{i}, u_{i+1}, \ldots, u_{m}\right) \\
& -N_{i}\left(v_{1}, \ldots, v_{i-1}, v_{i}, v_{i+1}, \ldots, v_{m}\right) \| \\
\leq & \sum_{j=1, j \neq i}^{m} \zeta_{i j}\left\|u_{j}-v_{j}\right\| .
\end{aligned}
$$

From (40)-(41), we obtain

$$
\begin{aligned}
\left\|P_{\rho_{i}}\left(u_{1}, u_{2}, \ldots, u_{m}\right)-P_{\rho_{i}}\left(v_{1}, v_{2}, \ldots, v_{m}\right)\right\| \\
\leq \frac{\rho_{i} \tau_{i}}{\delta_{i}} \sum_{j=1, j \neq i}^{m} \zeta_{i j}\left\|u_{j}-v_{j}\right\| \\
\quad+\frac{\tau_{i}}{\delta_{i}} \sqrt[q_{i}]{1-q_{i} \rho_{i} \sigma_{i}+c_{q_{i}} \rho_{i}^{q_{i}} \zeta_{i i}^{q_{i}}}\left\|u_{i}-v_{i}\right\|
\end{aligned}
$$

for $i=1,2, \ldots, m$. Equation (42) implies that

$$
\begin{aligned}
& \sum_{j=1}^{m}\left\|P_{\rho_{j}}\left(u_{1}, u_{2}, \ldots, u_{m}\right)-P_{\rho_{j}}\left(v_{1}, v_{2}, \ldots, v_{m}\right)\right\| \\
& =\sum_{i=1}^{m}\left\|P_{\rho_{i}}\left(u_{1}, u_{2}, \ldots, u_{m}\right)-P_{\rho_{i}}\left(v_{1}, v_{2}, \ldots, v_{m}\right)\right\| \\
& \leq \sum_{i=1}^{m}\left(\frac{\tau_{i}}{\delta_{i}} \sqrt[q_{i}]{1-q_{i} \rho_{i} \sigma_{i}+c_{q_{i}} \rho_{i}^{q_{i}} \zeta_{i i}^{q_{i}}}\left\|u_{i}-v_{i}\right\|\right. \\
& \left.+\frac{\rho_{i} \tau_{i}}{\delta_{i}} \sum_{j=1, j \neq i}^{m} \zeta_{i j}\left\|u_{j}-v_{j}\right\|\right) \\
& \leq \sum_{i=1}^{m} \frac{\tau_{i}}{\delta_{i}} \sqrt[q_{i}]{1-q_{i} \rho_{i} \sigma_{i}+c_{q_{i}} \rho_{i}^{q_{i}} \zeta_{i i}^{q_{i}}}\left\|u_{i}-v_{i}\right\| \\
& +\sum_{i=1}^{m} \frac{\rho_{i} \tau_{i}}{\delta_{i}} \sum_{j=1, j \neq i}^{m} \zeta_{i j}\left\|u_{j}-v_{j}\right\| \\
& =\sum_{j=1}^{m} \frac{\tau_{j}}{\delta_{j}} \sqrt[q_{j}]{1-q_{j} \rho_{j} \sigma_{j}+c_{q_{j}} \rho_{j}^{q_{j}} \zeta_{j j}^{q_{j}}}\left\|u_{j}-v_{j}\right\| \\
& +\sum_{j=1}^{m}\left(\sum_{i=1, i \neq j}^{m} \frac{\rho_{i} \tau_{i} \zeta_{i j}}{\delta_{i}}\right)\left\|u_{j}-v_{j}\right\| \\
& =\sum_{j=1}^{m}\left[\frac{\tau_{j}}{\delta_{j}} \sqrt[q_{j}]{1-q_{j} \rho_{j} \sigma_{j}+c_{q_{j}} \rho_{j}^{q_{j}} \zeta_{j j}^{q_{j}}}+\sum_{i=1, i \neq j}^{m} \frac{\rho_{i} \tau_{i} \zeta_{i j}}{\delta_{i}}\right] \\
& \times\left\|u_{j}-v_{j}\right\| \\
& \leq k \sum_{j=1}^{m}\left\|u_{j}-v_{j}\right\|
\end{aligned}
$$

where $k=\max _{1 \leq j \leq m}\left\{\left(\tau_{j} / \delta_{j}\right) \sqrt[q_{j}]{1-q_{j} \rho_{j} \sigma_{j}+c_{q_{j}} \rho_{j}^{q_{j}} \zeta_{j j}^{q_{j}}}+\right.$ $\left.\sum_{i=1, i \neq j}^{m}\left(\zeta_{i j} \rho_{i} \tau_{i} / \delta_{i}\right)\right\}$. By (33), we know that $0 \leq k<1$. It follows from (43) that

$$
\begin{aligned}
& \left\|F\left(u_{1}, u_{2}, \ldots, u_{m}\right)-F\left(v_{1}, v_{2}, \ldots, v_{m}\right)\right\|_{*} \\
& \quad \leq k\left\|\left(u_{1}, u_{2}, \ldots, u_{m}\right)-\left(v_{1}, v_{2}, \ldots, v_{m}\right)\right\|_{*} .
\end{aligned}
$$

This proves that $F: X_{1} \times X_{2} \times \cdots \times X_{m} \rightarrow X_{1} \times X_{2} \times \cdots \times$ $X_{m}$ is a contraction mapping. Hence, there exists a unique $\left(x_{1}^{*}, x_{2}^{*}, \ldots, x_{m}^{*}\right) \in X_{1} \times X_{2} \times \cdots \times X_{m}$ such that

$$
F\left(\left(x_{1}^{*}, x_{2}^{*}, \ldots, x_{m}^{*}\right)\right)=\left(x_{1}^{*}, x_{2}^{*}, \ldots, x_{m}^{*}\right) ;
$$

that is, $P_{\rho_{i}}\left(x_{1}^{*}, x_{2}^{*}, \ldots, x_{m}^{*}\right)=x_{i}^{*}$ for $i=1,2, \ldots, m$; that is,

$$
x_{i}^{*}=J_{M_{i}}^{\rho_{i}}\left[x_{i}^{*}-\rho_{i} N_{i}\left(x_{1}^{*}, x_{2}^{*}, \ldots, x_{m}^{*}\right)\right] .
$$

By Lemma $11,\left(x_{1}^{*}, x_{2}^{*}, \ldots, x_{m}^{*}\right)$ is the unique solution of problem (1). This completes the proof.

Remark 13. If $m=2$, then Theorem 12 reduces to Theorem 3.2 of Lan [19].

Corollary 14. Let $\mathscr{H}_{i}$ be real Hilbert space and $\eta_{i}: \mathscr{H}_{i} \times \mathscr{H}_{i} \rightarrow$ $\mathscr{H}_{i}$ be $\tau_{i}$-Lipschitz continuous and $\delta_{i}$-strongly monotone for any $i=1,2, \ldots, m$. Suppose that $M_{i}: \mathscr{H}_{i} \rightarrow 2^{\mathscr{H}_{i}}$ is maximal $\eta_{i}$-monotone mapping, $N_{i}: \mathscr{H}_{1} \times \mathscr{H}_{2} \times \cdots \times \mathscr{H}_{m} \rightarrow \mathscr{H}_{i}$ is $\sigma_{i}$-strongly monotone in the ith argument, and $\left(\zeta_{i 1}, \ldots, \zeta_{i i}, \ldots, \zeta_{i m}\right)$-Lipschitz continuous for $i=1,2, \ldots, m$. If

$$
\frac{\tau_{j}}{\delta_{j}} \sqrt[2]{1-2 \rho_{j} \sigma_{j}+\rho_{j}^{2} \zeta_{j j}^{2}}+\sum_{i=1, i \neq j}^{m} \frac{\zeta_{i j} \rho_{i} \tau_{i}}{\delta_{i}}<1,
$$

then problem (1) has a unique solution $\left(x_{1}^{*}, x_{2}^{*}, \ldots, x_{m}^{*}\right) \in$ $\mathscr{H}_{1} \times \mathscr{H}_{2} \times \cdots \times \mathscr{H}_{m}$.

Corollary 15. Let $\mathscr{H}_{i}$ be real Hilbert space for any $i=1,2, \ldots, m$. Suppose that $\varphi_{i}: \mathscr{H}_{i} \rightarrow(-\infty,+\infty]$ is proper, convex, and lower semicontinuous functional on $\mathscr{H}_{i}$ and $N_{i}: \mathscr{H}_{1} \times \mathscr{H}_{2} \times \cdots \times \mathscr{H}_{m} \rightarrow \mathscr{H}_{i}$ is $\sigma_{i}$-strongly monotone in the ith argument and $\left(\zeta_{i 1}, \ldots, \zeta_{i i}, \ldots, \zeta_{i m}\right)$-Lipschitz continuous for $i=1,2, \ldots, m$. If

$$
\sqrt[2]{1-2 \rho_{j} \sigma_{j}+\rho_{j}^{2} \zeta_{j j}^{2}}+\sum_{i=1, i \neq j}^{m} \rho_{i} \zeta_{i j}<1,
$$

then problem (2) has a unique solution $\left(x_{1}^{*}, x_{2}^{*}, \ldots, x_{m}^{*}\right) \in$ $\mathscr{H}_{1} \times \mathscr{H}_{2} \times \cdots \times \mathscr{H}_{m}$.

\section{Perturbed Iterative Algorithms}

In this section, by using Definition 9 and Lemma 10, we construct a new perturbed iterative algorithm with mixed errors for solving problem (1) and prove the convergence and stability of the iterative sequence generated by the algorithm.

Algorithm 16. Let $\eta_{i}: X_{i} \times X_{i} \rightarrow X_{i}^{*}$ and $N_{i}: X_{1} \times X_{2} \times \cdots \times$ $X_{m} \rightarrow X_{i}$ be single-valued mappings and let $M_{i}: X_{i} \rightarrow 2^{X_{i}}$ 
be generalized $m$-accretive mapping for $i=1,2, \ldots, m$. For any given initial point $\left(x_{1}^{0}, x_{2}^{0}, \ldots, x_{m}^{0}\right) \in X_{1} \times X_{2} \times \cdots \times X_{m}$, the perturbed iterative sequence $\left\{\left(x_{1}^{n}, x_{2}^{n}, \ldots, x_{m}^{n}\right)\right\}$ for problem (1) is defined by

$$
\begin{aligned}
x_{i}^{n+1}= & \left(1-\alpha_{n}\right) x_{i}^{n}+\alpha_{n} J_{M_{i}}^{\rho_{i}}\left[x_{i}^{n}-\rho_{i} N_{i}\left(x_{1}^{n}, x_{2}^{n}, \ldots, x_{m}^{n}\right)\right] \\
& +\alpha_{n} u_{i}^{n}+w_{i}^{n},
\end{aligned}
$$

where $n \geq 0, i=1,2, \ldots, m,\left\{\alpha_{n}\right\}$ is a sequence in $[0,1]$, and $\left\{u_{i}^{n}\right\},\left\{w_{i}^{n}\right\} \subset X_{i}$ are errors to take into account a possible inexact computation of the resolvent operator point satisfying the following conditions:

(i) $u_{i}^{n}=u_{i}^{\prime n}+u_{i}^{\prime \prime n}$;

(ii) $\lim _{n \rightarrow \infty}\left\|u_{i}^{\prime n}\right\|=0$;

(iii) $\sum_{n=0}^{\infty}\left\|u_{i}^{\prime \prime n}\right\|<+\infty, \sum_{n=0}^{\infty}\left\|w_{i}^{n}\right\|<+\infty$ for $i=$ $1,2, \ldots, m$.

Let $\left\{\left(z_{1}^{n}, z_{2}^{n}, \ldots, z_{m}^{n}\right)\right\}$ be any sequence in $X_{1} \times X_{2} \times \cdots \times X_{m}$ and define $\left\{\left(\epsilon_{1}^{n}, \epsilon_{2}^{n}, \ldots, \epsilon_{m}^{n}\right)\right\}$ by

$$
\begin{aligned}
\epsilon_{i}^{n}=\| z_{i}^{n+1}-\{(1 & \left.-\alpha_{n}\right) z_{i}^{n} \\
& +\alpha_{n} J_{M_{i}}^{\rho_{i}}\left[z_{i}^{n}-\rho_{i} N_{i}\left(z_{1}^{n}, z_{2}^{n}, \ldots, z_{m}^{n}\right)\right] \\
& \left.+\alpha_{n} u_{i}^{n}+w_{i}^{n}\right\} \|
\end{aligned}
$$

for $i=1,2, \ldots, m$.

Algorithm 17. Let $\eta_{i}: \mathscr{H}_{i} \times \mathscr{H}_{i} \rightarrow \mathscr{H}_{i}$ and $N_{i}: \mathscr{H}_{1} \times \mathscr{H}_{2} \times \cdots \times$ $\mathscr{H}_{m} \rightarrow \mathscr{H}_{i}$ be single-valued mappings and let $M_{i}: \mathscr{H}_{i} \rightarrow$ $2^{\mathscr{H}_{i}}$ be maximal $\eta_{i}$-monotone mapping for $i=1,2, \ldots, m$. For any given initial point $\left(x_{1}^{0}, x_{2}^{0}, \ldots, x_{m}^{0}\right) \in \mathscr{H}_{1} \times \mathscr{H}_{2} \times \cdots \times$ $\mathscr{H}_{m}$, the perturbed iterative sequence $\left\{\left(x_{1}^{n}, x_{2}^{n}, \ldots, x_{m}^{n}\right)\right\}$ for problem (1) is defined by

$$
\begin{aligned}
x_{i}^{n+1}= & \left(1-\alpha_{n}\right) x_{i}^{n}+\alpha_{n} J_{M_{i}}^{\rho_{i}}\left[x_{i}^{n}-\rho_{i} N_{i}\left(x_{1}^{n}, x_{2}^{n}, \ldots, x_{m}^{n}\right)\right] \\
& +\alpha_{n} u_{i}^{n}+w_{i}^{n},
\end{aligned}
$$

where $n \geq 0, i=1,2, \ldots, m,\left\{\alpha_{n}\right\}$ is a sequence in $[0,1]$, and $\left\{u_{i}^{n}\right\},\left\{w_{i}^{n}\right\} \subset X_{i}$ are errors to take into account a possible inexact computation of the resolvent operator point satisfying the following conditions:

(i) $\lim _{n \rightarrow \infty}\left\|u_{i}^{n}\right\|=0$;

(ii) $\sum_{n=0}^{\infty}\left\|w_{i}^{n}\right\|<+\infty$ for $i=1,2, \ldots, m$.

Let $\left\{\left(z_{1}^{n}, z_{2}^{n}, \ldots, z_{m}^{n}\right)\right\}$ be any sequence in $\mathscr{H}_{1} \times \mathscr{H}_{2} \times \cdots \times \mathscr{H}_{m}$ and define $\left\{\left(\epsilon_{1}^{n}, \epsilon_{2}^{n}, \ldots, \epsilon_{m}^{n}\right)\right\}$ by

$$
\begin{aligned}
\epsilon_{i}^{n}=\| z_{i}^{n+1}-\{(1- & \left.\alpha_{n}\right) z_{i}^{n} \\
& +\alpha_{n} J_{M_{i}}^{\rho_{i}}\left[z_{i}^{n}-\rho_{i} N_{i}\left(z_{1}^{n}, z_{2}^{n}, \ldots, z_{m}^{n}\right)\right] \\
& \left.+\alpha_{n} u_{i}^{n}+w_{i}^{n}\right\} \|
\end{aligned}
$$

for $i=1,2, \ldots, m$.
Algorithm 18. Let $N_{i}: \mathscr{H}_{1} \times \mathscr{H}_{2} \times \cdots \times \mathscr{H}_{m} \rightarrow \mathscr{H}_{i}$ be single-valued mappings and $\varphi_{i}: \mathscr{H}_{i} \rightarrow(-\infty,+\infty]$ is proper, convex, and lower semi-continuous functional on $\mathscr{H}_{i}$ for $i=1,2, \ldots, m$. For any given initial point $\left(x_{1}^{0}, x_{2}^{0}, \ldots, x_{m}^{0}\right) \in$ $\mathscr{H}_{1} \times \mathscr{H}_{2} \times \cdots \times \mathscr{H}_{m}$, the perturbed iterative sequence $\left\{\left(x_{1}^{n}, x_{2}^{n}, \ldots, x_{m}^{n}\right)\right\}$ for problem (2) is defined by

$$
x_{i}^{n+1}=\left(1-\alpha_{n}\right) x_{i}^{n}+\alpha_{n} J_{\varphi_{i}}\left[x_{i}^{n}-\rho_{i} N_{i}\left(x_{1}^{n}, x_{2}^{n}, \ldots, x_{m}^{n}\right)\right]+w_{i}^{n},
$$

where $n \geq 0, i=1,2, \ldots, m,\left\{\alpha_{n}\right\}$ is a sequence in $[0,1]$, $\left\{w_{i}^{n}\right\} \subset X_{i}$ are errors to take into account a possible inexact computation of the resolvent operator point satisfying the condition $\quad \sum_{n=0}^{\infty}\left\|w_{i}^{n}\right\|<+\infty$ for $i=1,2, \ldots, m$. Let $\left\{\left(z_{1}^{n}, z_{2}^{n}, \ldots, z_{m}^{n}\right)\right\}$ be any sequence in $\mathscr{H}_{1} \times \mathscr{H}_{2} \times \cdots \times \mathscr{H}_{m}$ and define $\left\{\left(\epsilon_{1}^{n}, \epsilon_{2}^{n}, \ldots, \epsilon_{m}^{n}\right)\right\}$ by

$$
\begin{aligned}
\epsilon_{i}^{n}=\| z_{i}^{n+1}-\{( & \left(-\alpha_{n}\right) z_{i}^{n} \\
& +\alpha_{n} J_{\varphi_{i}}\left[z_{i}^{n}-\rho_{i} N_{i}\left(z_{1}^{n}, z_{2}^{n}, \ldots, z_{m}^{n}\right)\right] \\
& \left.+w_{i}^{n}\right\} \|
\end{aligned}
$$

for $i=1,2, \ldots, m$.

Remark 19. If $m=2$, then Algorithm 16 reduces to Algorithm 4.3 of Lan [19].

Next we will show the convergence and stability of Algorithm 16.

Theorem 20. Suppose that $X_{i}, \eta_{i}, N_{i}$, and $M_{i}(i=1,2, \ldots, m)$ are the same as in Theorem 12. If $\sum_{n=0}^{\infty} \alpha_{n}=+\infty$ and condition (33) holds, then the perturbed iterative sequence $\left\{\left(x_{1}^{n}, x_{2}^{n}, \ldots, x_{m}^{n}\right)\right\}$ defined by Algorithm 16 converges strongly to the unique solution $\left(x_{1}^{*}, x_{2}^{*}, \ldots, x_{m}^{*}\right) \in X_{1} \times X_{2} \times \cdots \times X_{m}$ of the problem (1). Moreover, if there exists $a \in\left(0, \alpha_{n}\right]$ for all $n \geq 0$, then

$$
\lim _{n \rightarrow \infty}\left(z_{1}^{n}, z_{2}^{n}, \ldots, z_{m}^{n}\right)=\left(x_{1}^{*}, x_{2}^{*}, \ldots, x_{m}^{*}\right)
$$

if and only if

$$
\lim _{n \rightarrow \infty}\left(\epsilon_{1}^{n}, \epsilon_{2}^{n}, \ldots, \epsilon_{m}^{n}\right)=\underbrace{(0,0, \ldots, 0)}_{m}
$$

where $\left(\epsilon_{1}^{n}, \epsilon_{2}^{n}, \ldots, \epsilon_{m}^{n}\right)$ is defined by (50).

Proof. From Theorem 12, we know that problem (1) has a unique solution

$$
\left(x_{1}^{*}, x_{2}^{*}, \ldots, x_{m}^{*}\right) \in X_{1} \times X_{2} \times \cdots \times X_{m} .
$$


It follows from (49) and the proof of (42) in Theorem 12 that, for $i=1,2, \ldots, m$,

$$
\begin{aligned}
& \left\|x_{i}^{n+1}-x_{i}^{*}\right\| \\
& \leq\left(1-\alpha_{n}\right)\left\|x_{i}^{n}-x_{i}^{*}\right\| \\
& +\alpha_{n}\left\{\frac{\tau_{i}}{\delta_{i}} \sqrt[q_{i}]{1-q_{i} \rho_{i} \sigma_{i}+c_{q_{i}} \rho_{i}^{q_{i}} \zeta_{i i}^{q_{i}}}\left\|x_{i}^{n}-x_{i}^{*}\right\|\right. \\
& \left.+\frac{\rho_{i} \tau_{i}}{\delta_{i}} \sum_{j=1, j \neq i}^{m} \zeta_{i j}\left\|x_{j}^{n}-x_{j}^{*}\right\|\right\} \\
& +\alpha_{n}\left\|u_{i}^{\prime n}\right\|+\left(\left\|u_{i}^{\prime \prime n}\right\|+\left\|w_{i}^{n}\right\|\right) .
\end{aligned}
$$

It follows from (58), we have

$$
\begin{aligned}
& \sum_{i=1}^{m}\left\|x_{i}^{n+1}-x_{i}^{*}\right\| \\
& \leq\left(1-\alpha_{n}\right) \sum_{i=1}^{m}\left\|x_{i}^{n}-x_{i}^{*}\right\| \\
& \quad+\alpha_{n} \sum_{j=1}^{m}\left[\frac{\tau_{j}}{\delta_{j}} \sqrt[q_{j}]{1-q_{j} \rho_{j} \sigma_{j}+c_{q_{j}} \rho_{j}^{q_{j}} \zeta_{j j}^{q_{j}}}+\sum_{i=1, i \neq j}^{m} \frac{\rho_{i} \tau_{i} \zeta_{i j}}{\delta_{i}}\right] \\
& \quad \times\left\|x_{j}^{n}-x_{j}^{*}\right\|+\alpha_{n} \sum_{i=1}^{m}\left\|u_{i}^{\prime n}\right\|+\sum_{i=1}^{m}\left\|u_{i}^{\prime \prime n}\right\|+\sum_{i=1}^{m}\left\|w_{i}^{n}\right\| \\
& =\left(1-\alpha_{n}\right) \sum_{j=1}^{m}\left\|x_{j}^{n}-x_{j}^{*}\right\| \\
& +\alpha_{n} \sum_{j=1}^{m}\left[\frac{\tau_{j}}{\delta_{j}} \sqrt[q_{j}]{1-q_{j} \rho_{j} \sigma_{j}+c_{q_{j}} \rho_{j}^{q_{j}} \zeta_{j j}^{q_{j}}}+\sum_{i=1, i \neq j}^{m} \frac{\rho_{i} \tau_{i} \zeta_{i j}}{\delta_{i}}\right] \\
& \quad \times\left\|x_{j}^{n}-x_{j}^{*}\right\|+\alpha_{n} \sum_{j=1}^{m}\left\|u_{j}^{\prime n}\right\|+\sum_{j=1}^{m}\left\|u_{j}^{\prime \prime n}\right\|+\sum_{j=1}^{m}\left\|w_{j}^{n}\right\| \\
& \quad
\end{aligned}
$$

where $k$ is the same as in (43). Letting $t_{n}=$ $\alpha_{n}(1-k) \in[0,1], b_{n}=(1 /(1-k)) \sum_{j=1}^{m}\left\|u_{j}^{\prime n}\right\|$, and $c_{n}=\sum_{j=1}^{m}\left\|u_{j}^{\prime \prime n}\right\|+\sum_{j=1}^{m}\left\|w_{j}^{n}\right\|(n \geq 0)$, then it follows from $\sum_{n=0}^{\infty} \alpha_{n}=+\infty$ and (i)-(iii) of Algorithm 16 that

$$
\begin{gathered}
\sum_{n=0}^{\infty} t_{n}=+\infty, \quad \lim _{n \rightarrow \infty} b_{n}=\frac{1}{1-k} \sum_{j=1}^{m}\left(\lim _{n \rightarrow \infty}\left\|u_{j}^{\prime n}\right\|\right)=0, \\
\sum_{n=0}^{\infty} c_{n}=\sum_{j=1}^{m} \sum_{n=0}^{\infty}\left\|u_{j}^{\prime \prime n}\right\|+\sum_{j=1}^{m} \sum_{n=0}^{\infty}\left\|w_{j}^{n}\right\|<+\infty .
\end{gathered}
$$

Setting $a_{n}=\sum_{j=1}^{m}\left\|x_{j}^{n}-x_{j}^{*}\right\|$, then (59) can be rewritten as

$$
a_{n+1} \leq\left(1-t_{n}\right) a_{n}+b_{n} t_{n}+c_{n}, \quad n=0,1,2, \ldots
$$

It follows from Lemma 10 that $\lim _{n \rightarrow \infty} a_{n}=0$; that is,

$$
\lim _{n \rightarrow \infty} \sum_{j=1}^{m}\left\|x_{j}^{n}-x_{j}^{*}\right\|=0 ;
$$

thus

$$
x_{j}^{n} \longrightarrow x_{j}^{*} \quad(n \longrightarrow \infty),(j=1,2, \ldots, m) .
$$

Hence, we know that the sequence $\left\{\left(x_{1}^{n}, x_{2}^{n}, \ldots, x_{m}^{n}\right)\right\}$ converges strongly to the unique solution $\left(x_{1}^{*}, x_{2}^{*}, \ldots, x_{m}^{*}\right)$ of the problem (1).

Now we prove the second conclusion. By (50), now we know

$$
\begin{aligned}
& \left\|z_{i}^{n+1}-x_{i}^{*}\right\| \\
& \leq \|\left(1-\alpha_{n}\right) z_{i}^{n}+\alpha_{n} J_{M_{i}}^{\rho_{i}}\left[z_{i}^{n}-\rho_{i} N_{i}\left(z_{1}^{n}, z_{2}^{n}, \ldots, z_{m}^{n}\right)\right] \\
& \quad+\alpha_{n} u_{i}^{n}+w_{i}^{n}-x_{i}^{*} \|+\epsilon_{i}^{n},
\end{aligned}
$$

where $i=1,2, \ldots, m$. As the proof of inequality (59), we have

$$
\begin{aligned}
& \sum_{j=1}^{m} \|\left(1-\alpha_{n}\right) z_{j}^{n}+\alpha_{n} J_{M_{j}}^{\rho_{j}}\left[z_{j}^{n}-\rho_{j} N_{j}\left(z_{1}^{n}, z_{2}^{n}, \ldots, z_{m}^{n}\right)\right] \\
& \quad+\alpha_{n} u_{j}^{n}+w_{j}^{n}-x_{j}^{*} \| \\
& \leq\left[1-\alpha_{n}(1-k)\right] \sum_{j=1}^{m}\left\|z_{j}^{n}-x_{j}^{*}\right\| \\
& +\alpha_{n}(1-k) \cdot \frac{1}{1-k} \sum_{j=1}^{m}\left\|u_{j}^{\prime n}\right\| \\
& +\left(\sum_{j=1}^{m}\left\|u_{j}^{\prime \prime n}\right\|+\sum_{j=1}^{m}\left\|w_{j}^{n}\right\|\right) .
\end{aligned}
$$


Since $0<a \leq \alpha_{n}(n=0,1,2, \ldots$,$) , it follows from (64) and$ (65) that

$$
\begin{aligned}
& \sum_{j=1}^{m}\left\|z_{j}^{n+1}-x_{j}^{*}\right\| \\
& \leq\left[1-\alpha_{n}(1-k)\right] \sum_{j=1}^{m}\left\|z_{j}^{n}-x_{j}^{*}\right\| \\
& +\alpha_{n}(1-k) \cdot \frac{1}{1-k}\left(\sum_{j=1}^{m}\left\|u_{j}^{\prime n}\right\|+\frac{1}{a} \sum_{j=1}^{m} \epsilon_{j}^{n}\right) \\
& +\left(\sum_{j=1}^{m}\left\|u_{j}^{\prime \prime n}\right\|+\sum_{j=1}^{m}\left\|w_{j}^{n}\right\|\right) .
\end{aligned}
$$

Suppose that $\lim _{n \rightarrow \infty}\left(\epsilon_{1}^{n}, \epsilon_{2}^{n}, \ldots, \epsilon_{m}^{n}\right)=\underbrace{(0,0, \ldots, 0)}_{m}$. Letting $b_{n}^{\prime}=(1 /(1-k))\left(\sum_{j=1}^{m}\left\|u_{j}^{\prime n}\right\|+(1 / a) \sum_{j=1}^{m} \epsilon_{j}^{n}\right)$ and $a_{n}^{\prime}=\sum_{j=1}^{m} \| z_{j}^{n}-$ $x_{j}^{*} \|$, then (66) implies that

$$
a_{n+1}^{\prime} \leq\left(1-t_{n}\right) a_{n}^{\prime}+b_{n}^{\prime} t_{n}+c_{n}, \quad n=0,1,2, \ldots,
$$

where $t_{n}, c_{n}$ are the same as previously. Since $\lim _{n \rightarrow \infty}\left\|u_{j}^{\prime n}\right\|=$ 0 and $\lim _{n \rightarrow \infty} \epsilon_{j}^{n}=0 \quad(j=1,2, \ldots, m)$,

$$
\lim _{n \rightarrow \infty} b_{n}^{\prime}=\frac{1}{1-k}\left[\sum_{j=1}^{m}\left(\lim _{n \rightarrow \infty}\left\|u_{j}^{\prime n}\right\|\right)+\frac{1}{a} \sum_{j=1}^{m}\left(\lim _{n \rightarrow \infty} \epsilon_{j}^{n}\right)\right]=0 .
$$

It again follows from Lemma 10, we have $\lim _{n \rightarrow \infty} a_{n}^{\prime}=0$ and so

$$
\lim _{n \rightarrow \infty}\left(z_{1}^{n}, z_{2}^{n}, \ldots, z_{m}^{n}\right)=\left(x_{1}^{*}, x_{2}^{*}, \ldots, x_{m}^{*}\right) .
$$

Conversely, if $\lim _{n \rightarrow \infty}\left(z_{1}^{n}, z_{2}^{n}, \ldots, z_{m}^{n}\right)=\left(x_{1}^{*}, x_{2}^{*}, \ldots, x_{m}^{*}\right)$, it follows from (50), then we get

$$
\begin{aligned}
\epsilon_{i}^{n} \leq & \left\|z_{i}^{n+1}-x_{i}^{*}\right\| \\
& +\|\left(1-\alpha_{n}\right) z_{i}^{n}+\alpha_{n} J_{M_{j}}^{\rho_{i}}\left[z_{i}^{n}-\rho_{i} N_{i}\left(z_{1}^{n}, z_{2}^{n}, \ldots, z_{m}^{n}\right)\right] \\
& +\alpha_{n} u_{i}^{n}+w_{i}^{n}-x_{i}^{*} \|, \quad \forall i=1,2, \ldots, m
\end{aligned}
$$

Combining (65) with (70), we have

$$
\begin{aligned}
\sum_{i=1}^{m} \epsilon_{i}^{n} \leq & \sum_{i=1}^{m}\left\|z_{i}^{n+1}-x_{i}^{*}\right\|+\left[1-\alpha_{n}(1-k)\right] \sum_{j=1}^{m}\left\|z_{j}^{n}-x_{j}^{*}\right\| \\
& +\alpha_{n}(1-k) \cdot \frac{1}{1-k} \sum_{j=1}^{m}\left\|u_{j}^{\prime n}\right\| \\
& +\left(\sum_{j=1}^{m}\left\|u_{j}^{\prime \prime n}\right\|+\sum_{j=1}^{m}\left\|w_{j}^{n}\right\|\right) \longrightarrow 0 \quad(n \longrightarrow \infty) .
\end{aligned}
$$

This completes the proof.
Corollary 21. Suppose that $\mathscr{H}_{i}, \eta_{i}, N_{i}$, and $M_{i}(i=$ $1,2, \ldots, m)$ are the same as in Corollary 14. If $\sum_{n=0}^{\infty} \alpha_{n}=+\infty$ and condition (47) holds, then the perturbed iterative sequence $\left\{\left(x_{1}^{n}, x_{2}^{n}, \ldots, x_{m}^{n}\right)\right\}$ defined by Algorithm 17 converges strongly to the unique solution $\left(x_{1}^{*}, x_{2}^{*}, \ldots, x_{m}^{*}\right) \in \mathscr{H}_{1} \times \mathscr{H}_{2} \times \cdots \times \mathscr{H}_{m}$ of the problem (1). Moreover, if there exists a $\in\left(0, \alpha_{n}\right]$ for all $n \geq 0$, then $\lim _{n \rightarrow \infty}\left(z_{1}^{n}, z_{2}^{n}, \ldots, z_{m}^{n}\right)=\left(x_{1}^{*}, x_{2}^{*}, \ldots, x_{m}^{*}\right)$ if and only if

$$
\lim _{n \rightarrow \infty}\left(\epsilon_{1}^{n}, \epsilon_{2}^{n}, \ldots, \epsilon_{m}^{n}\right)=\underbrace{(0,0, \ldots, 0)}_{m},
$$

where $\left(\epsilon_{1}^{n}, \epsilon_{2}^{n}, \ldots, \epsilon_{m}^{n}\right)$ is defined by (50).

Corollary 22. Assume that $\mathscr{H}_{i}, N_{i}$, and $\varphi_{i}(i=1,2, \ldots, m)$ are the same as in Corollary 15. If $\sum_{n=0}^{\infty} \alpha_{n}=+\infty$ and condition (48) holds, then the perturbed iterative sequence $\left\{\left(x_{1}^{n}, x_{2}^{n}, \ldots, x_{m}^{n}\right)\right\}$ defined by Algorithm 18 converges strongly to the unique solution $\left(x_{1}^{*}, x_{2}^{*}, \ldots, x_{m}^{*}\right) \in \mathscr{H}_{1} \times \mathscr{H}_{2} \times \cdots \times \mathscr{H}_{m}$ of the problem (2). Moreover, if there exists $a \in\left(0, \alpha_{n}\right]$ for all $n \geq 0$, then $\lim _{n \rightarrow \infty}\left(z_{1}^{n}, z_{2}^{n}, \ldots, z_{m}^{n}\right)=\left(x_{1}^{*}, x_{2}^{*}, \ldots, x_{m}^{*}\right)$ if and only if

$$
\lim _{n \rightarrow \infty}\left(\epsilon_{1}^{n}, \epsilon_{2}^{n}, \ldots, \epsilon_{m}^{n}\right)=\underbrace{(0,0, \ldots, 0)}_{m},
$$

where $\left(\epsilon_{1}^{n}, \epsilon_{2}^{n}, \ldots, \epsilon_{m}^{n}\right)$ is defined by (54).

Remark 23. If $m=2$, then Theorem 20 reduces to Theorem 4.3 of Lan [19]. Further, one can easily see that our results presented in this paper may be viewed as an refinement and improvement of the previously known results.

\section{Conflict of Interests}

The authors declare that there is no conflict of interests regarding the publication of this paper.

\section{Acknowledgments}

This work has been partially supported by Sichuan Province Cultivation Fund Project of academic and technical Leaders, the Open Research Fund of Key Laboratory of Higher Education of Sichuan Province for Enterprise Informationalization and Internet of Things (2013WZJ01), and the Cultivation Project of Sichuan University of Science and Engineering (2011PY01).

\section{References}

[1] R. P. Agarwal, Y. J. Cho, J. Li, and N. J. Huang, "Stability of iterative procedures with errors approximating common fixed points for a couple of quasi-contractive mapping in $q$-uniformly smooth Banach Spaces," Journal of Mathematical Analysis and Applications, vol. 272, no. 2, pp. 435-447, 2002.

[2] R. P. Agarwal, J. W. Chen, Y. J. Cho, and Z. P. Wan, "Stability analysis for parametric generalized vector quasi-variational-like inequality problems," Journal of Inequalities and Applications, vol. 2012, article 57, 2012.

[3] M. Alimohammady and M. Roohi, "A system of generalized variational inclusion problems involving $(A, \eta)$-monotone mappings," Filomat, vol. 23, no. 1, pp. 13-20, 2009. 
[4] X. P. Ding, "General algorithm of solutions for nonlinear variational inequalities in Banach space," Computers \& Mathematics with Applications, vol. 34, no. 9, pp. 131-137, 1997.

[5] X. P. Ding, "Iterative process with errors to locally strictly pseudocontractive maps in Banach spaces," Computers \& Mathematics with Applications, vol. 32, no. 10, pp. 91-97, 1996.

[6] X. P. Ding, "Perturbed proximal point algorithms for generalized quasivariational inclusions," Journal of Mathematical Analysis and Applications, vol. 210, no. 1, pp. 88-101, 1997.

[7] Y. P. Fang, N. J. Huang, and H. B. Thompson, "A new system of variational inclusions with $(H, \eta)$-monotone operators in Hilbert spaces," Computers \& Mathematics with Applications, vol. 49, no. 2-3, pp. 365-374, 2005.

[8] Y. P. Fang and N. J. Huang, " $H$-monotone operator and resolvent operator technique for variational inclusion," Applied Mathematics and Computation, vol. 145, no. 2-3, pp. 795-803, 2003.

[9] F. Gürsoy, V. Karakaya, and B. E. Rhoades, "Some convergence and stability results for the Kirk multistep and Kirk-SP fixed point iterative algorithms," Abstract and Applied Analysis, vol. 2014, Article ID 806537, 12 pages, 2014.

[10] N. J. Huang and Y. P. Fang, "Generalized $m$-accretive mappings in Banach spaces," Journal of Sichuan University, vol. 38, no. 4, pp. 591-592, 2001.

[11] N. J. Huang, Y. P. Fang, and C. X. Deng, "Nonlinear variational inclusions involving generalized $m$-accretive mappings," in Preceedings of the Bellman Continum: International Workshop on Uncertain Systems and Soft Computing, pp. 323-327, Beijing, China, 2002.

[12] J. U. Jeong, "Sensitivity analysis for a system of extended generalized nonlinear quasi-variational inclusions in $q$-uniformly smooth Banach spaces," International Mathematical Forum, vol. 7, no. 3, pp. 2465-2480, 2012.

[13] M. M. Jin, "Iterative algorithm for a new system of nonlinear setvalued variational inclusions involving $(H, \eta)$-monotone mappings," Journal of Inequalities in Pure and Applied Mathematics, vol. 7, no. 3, article 114, 2006.

[14] M. M. Jin, "A new system of general nonlinear variational inclusions involving $(A, \eta)$-accretive mappings in Banach Spaces," Mathematical Inequalities and Applications, vol. 11, no. 4, pp. 783-794, 2008.

[15] M. M. Jin, "Perturbed iterative algorithms for generalized nonlinear set-valued quasivariational inclusions involving generalized $m$-accretive mappings," Journal of Inequalities and Applications, vol. 2007, Article ID 29863, 12 pages, 2007.

[16] M. M. Jin, "Perturbed algorithm and stability for strongly nonlinear quasi-variational inclusion involving $H$-accretive operators," Mathematical Inequalities \& Applications, vol. 9, no. 4, pp. 771-779, 2006.

[17] K. R. Kazmi and M. I. Bhat, "Iterative algorithm for a system of nonlinear variational-like inclusions," Computers \& Mathematics with Applications, vol. 48, no. 12, pp. 1929-1935, 2004.

[18] K. R. Kazmi and M. I. Bhat, "Convergence and stability of iterative algorithms of generalized set-valued variationallike inclusions in Banach spaces," Applied Mathematics and Computation, vol. 166, no. 1, pp. 164-180, 2005.

[19] H. Y. Lan, "Stability of perturbed iterative algorithm for solving a system of generalized nonlinear equations," Nonlinear Functional Analysis and Applications, vol. 14, no. 1, pp. 1-11, 2009.
[20] H. Y. Lan and Q. K. Liu, "Iterative approximation for a system of nonlinear variational inclusions involving generalized $m$ accretive mappings," Nonlinear Analysis Forum, vol. 9, no. 1, pp. 33-42, 2004.

[21] H. Y. Lan and J. K. Kim, "Stable perturbed iteration procedures for solving new strongly nonlinear operator inclusions in Banach spaces," Nonlinear Functional Analysis and Application, vol. 18, no. 3, pp. 433-444, 2013.

[22] H. G. Li, "A nonlinear inclusion problem involving $(\alpha, \lambda)$ NODM set-valued mappings in ordered Hilbert space," Applied Mathematics Letters, vol. 25, no. 10, pp. 1384-1388, 2012.

[23] H. G. Li, D. Qiu, J. M. Zheng, and M. M. Jin, "Perturbed Ishikawa-hybrid quasi-proximal point algorithm with accretive mappings for a fuzzy system," Fixed Point Theory and Applications, vol. 2013, article 281, 2013.

[24] H. G. Li, D. Qiu, and M. M. Jin, "GNM ordered variational inequality system with ordered Lipschitz continuous mappings in an ordered Banach space," Journal of Inequalities and Applications, vol. 2013, article 514, 2013.

[25] H. G. Li, D. Qiu, and Y. Zou, "Characterizations of weakANODD set-valued mappings with applications to an approximate solution of GNMOQV inclusions involving $\oplus$ operator in ordered Banach spaces," Fixed Point Theory and Applications, vol. 2013, article 241, 2013.

[26] H. G. Li, A. J. Xu, and M. M. Jin, "A Hybrid proximal point three-step algorithm for nonlinear set-valued quasi-variational inclusions system involving $(A, \eta)$-accretive mappings," Fixed Point Theory and Applications, vol. 2010, Article ID 635382, 2010.

[27] H. G. Li, A. J. Xu, and M. M. Jin, "An Ishikawa-hybrid proximal point algorithm for nonlinear set-valued inclusions problem based on $(A, \eta)$-accretive framework," Fixed Point Theory and Applications, vol. 2010, Article ID 501293, 12 pages, 2010.

[28] Z. Q. Liu, J. S. Ume, and S. M. Kang, "On a system of nonlinear variational inclusions with $H_{h, n}$-monotone operators," Abstract and Applied Analysis, vol. 2012, Article ID 643828, 21 pages, 2012.

[29] J. W. Peng and L. J. Zhao, "General system of A-monotone nonlinear variational inclusions problems with applications," Journal of Inequalities and Applications, vol. 2009, Article ID 364615, 13 pages, 2009.

[30] P. Sunthrayuth and P. Kumam, "Iterative algorithms approach to a general system of nonlinear variational inequalities with perturbed mappings and fixed point problems for nonexpansive semigroups," Journal of Inequalities and Applications, vol. 2012, article 133, 2012.

[31] S. Saewan and P. Kumam, "Existence and algorithm for solving the system of mixed variational inequalities in Banach spaces," Journal of Applied Mathematics, vol. 2012, Article ID 413468, 15 pages, 2012.

[32] M. J. Shang, X. F. Su, and X. L. Qin, "An iterative method for a variational inequality and a fixed point problem for nonexpansive mappings," Acta Mathematica Scientia A, vol. 30, no. 4, pp. 1126-1137, 2010.

[33] Y. C. Xu, X. F. He, Z. B. Hou, and Z. He, "Generalized projection methods for Noor variational inequalities in Banach spaces," Acta Mathematica Scientia Shuxue Wuli Xuebao: Chinese Edition, vol. 30, no. 3, pp. 808-817, 2010.

[34] J. H. Zhu, S. S. Chang, and M. Liu, "Algorithms for a system of general variational inequalities in Banach spaces," Journal of Applied Mathematics, vol. 2012, Article ID 580158, 18 pages, 2012. 
[35] H. K. Xu, "Inequalities in Banach spaces with applications," Nonlinear Analysis: Theory, Methods \& Applications, vol. 16, no. 12, pp. 1127-1138, 1991.

[36] L. S. Liu, "Ishikawa and Mann iterative process with errors for nonlinear strongly accretive mappings in Banach spaces," Journal of Mathematical Analysis and Applications, vol. 194, no. 1, pp. 114-125, 1995. 


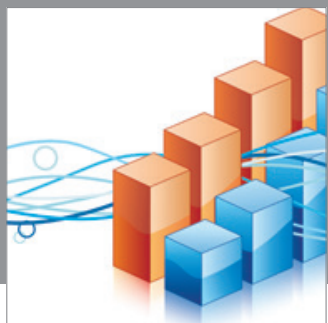

Advances in

Operations Research

mansans

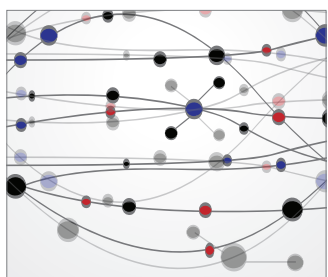

The Scientific World Journal
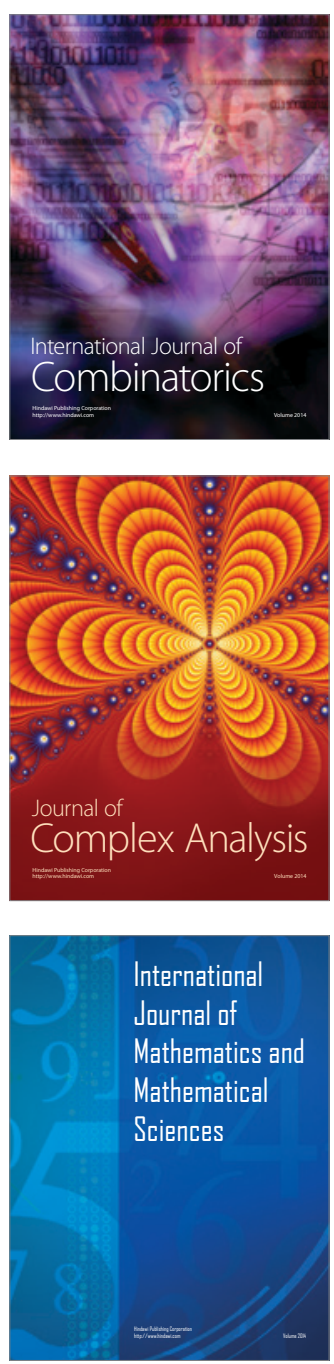
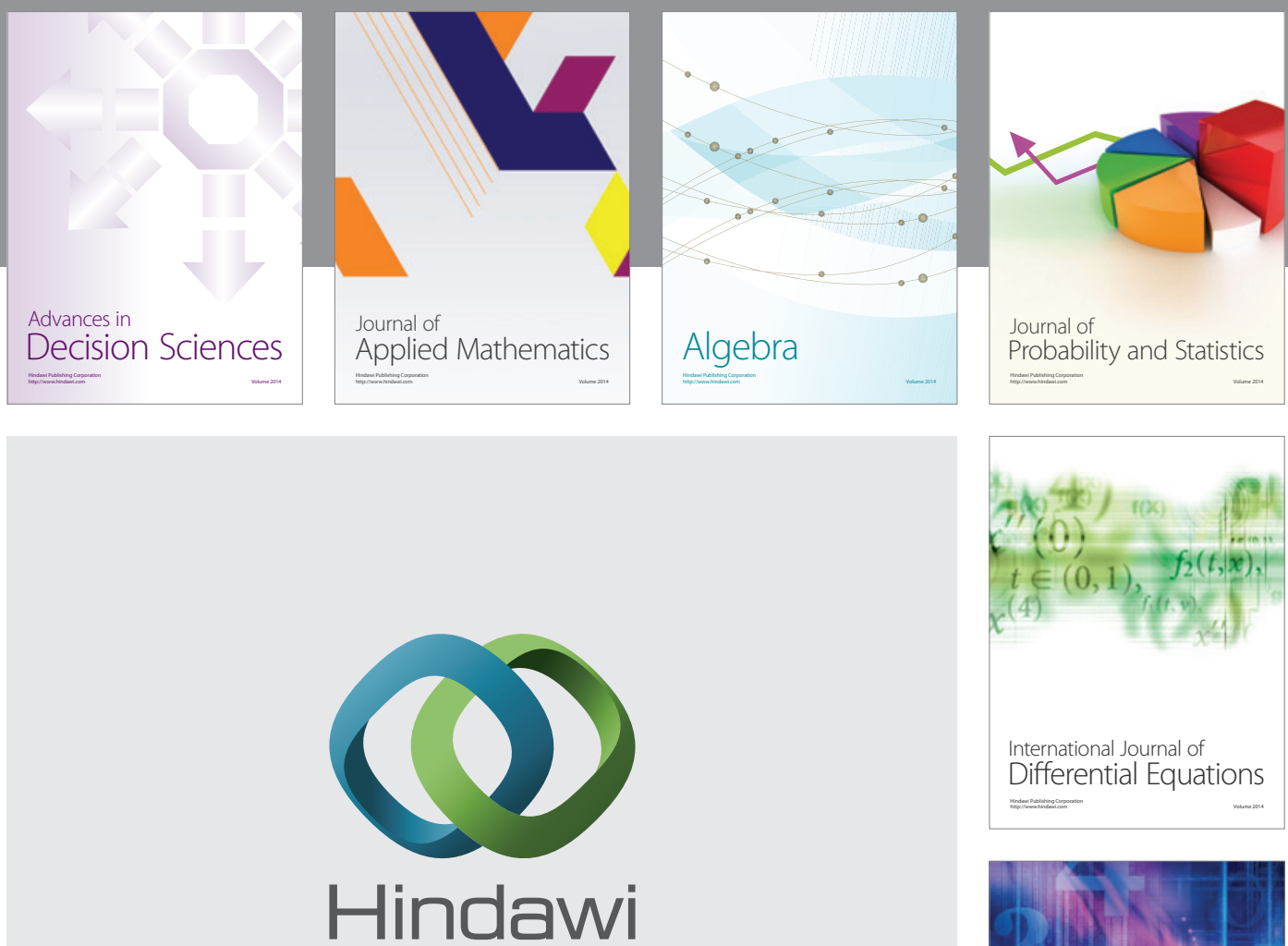

Submit your manuscripts at http://www.hindawi.com
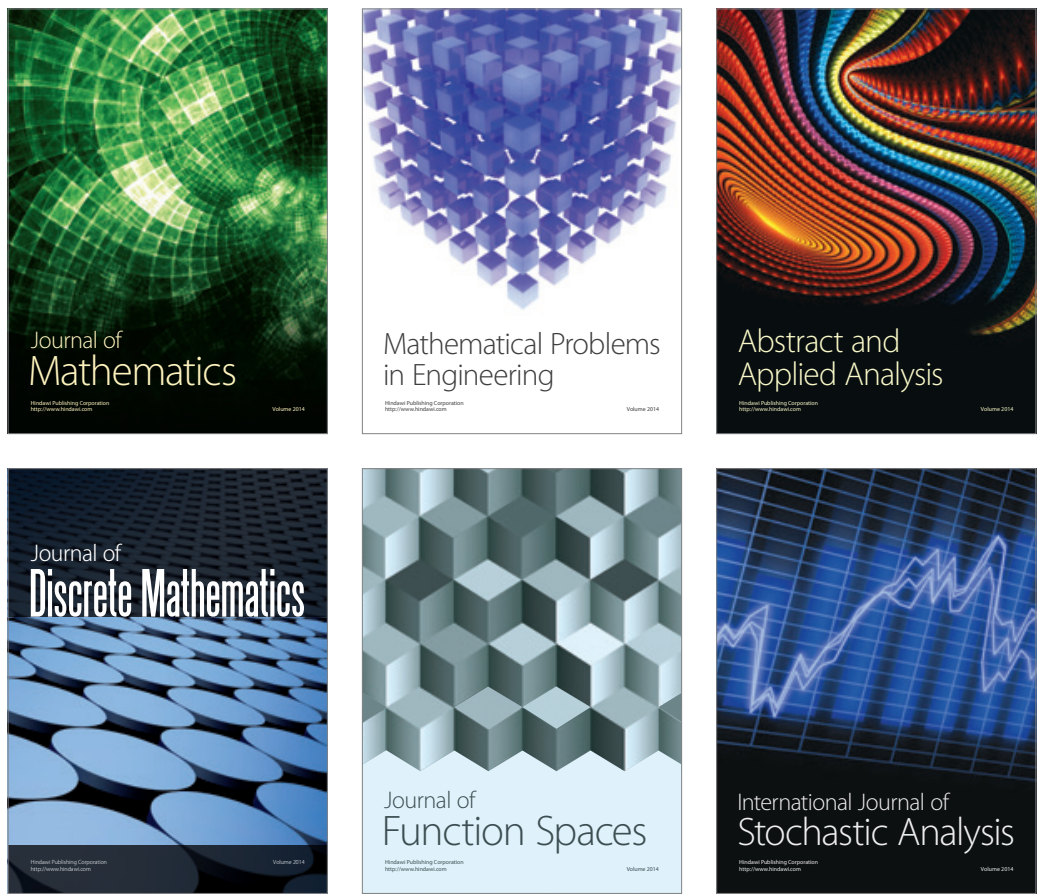

Journal of

Function Spaces

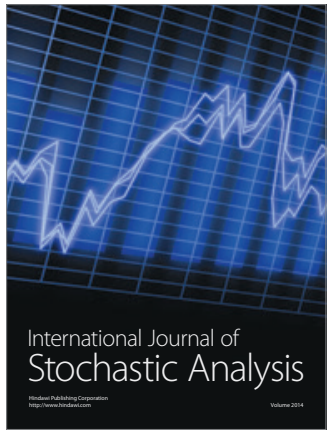

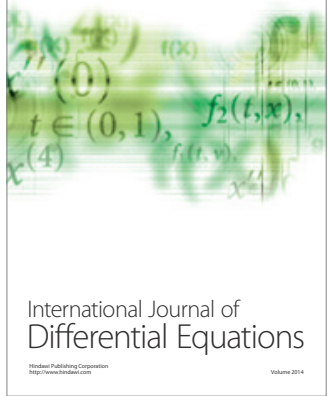
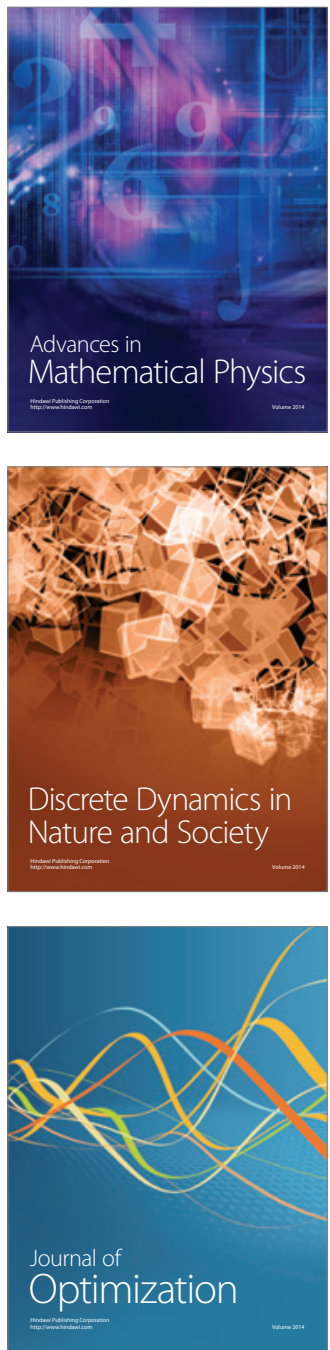